\title{
Bayesian Bhattacharyya bound for discrete-time filtering revisited
}

Carsten Fritsche and Fredrik Gustafsson

\section{Book Chapter}

N.B.: When citing this work, cite the original article.

Part of: Proc. of 2017 IEEE International Workshop on Computational Advances in Multi-Sensor Adaptive Processing (CAMSAP), 2017, pp. 719-723.

ISBN: 9781538612514

DOI: https:// doi.org/ 10.1109/CAMSAP.2017.8313201

Copyright: IEEE

Available at: Linköping University Institutional Repository (DiVA)

http:// urn.kb.se/ resolve?urn=urn:nbn:se:liu:diva-144022 


\title{
Bayesian Bhattacharyya Bound for discrete-time filtering revisited
}

\author{
Carsten Fritsche and Fredrik Gustafsson \\ Department of Electrical Engineering, Division of Automatic Control, Linköping University, Sweden \\ Email: firstname.lastname@liu.se
}

\begin{abstract}
In this paper, the derivation of the Bayesian Bhattacharyya bound for discrete-time filtering as proposed in a paper by Reece and Nicholson is revisited. It turns out that the results presented in the aforementioned contribution are incorrect, as some expectations appearing in the information matrix recursions are missing. This paper gives a generalized derivation of the $N$-th order Bayesian Bhattacharyya bound and presents corrected expressions for the case $N=2$. A nonlinear toy example is used to illustrate the results.
\end{abstract}

\section{INTRODUCTION}

Assessing the performance limits of any estimator is of great importance both practically and theoretically. In practice, it is desired to know the achievable accuracy limit of the application under investigation before implementing any estimator. In theory, the ultimate goal is to find a performance limit (or lower bound) that is tight, i.e. close to the performance of the optimal (in mean-square error (MSE) sense) estimator. In the context of discrete-time filtering, a couple of recursive lower bounds on the MSE have been derived that differ from each other in terms of implementation complexity, tightness, etc., see e.g. [1]-[5]. Among these, the perhaps most prominent example is the Bayesian Cramér-Rao bound derived by Tichavský et. al [1] which is easy to implement, but which in many situations yield a bound that is not tight [5]. In this paper, the Bayesian Bhattacharyya bound is revisited which is generally tighter or equal to the Bayesian Cramér-Rao bound, and which has an increased (but in many cases affordable) implementation complexity.

Consider the following discrete-time nonlinear system

$$
\begin{aligned}
& \mathbf{x}_{k}=\mathbf{f}_{k}\left(\mathbf{x}_{k-1}, \mathbf{v}_{k}\right), \\
& \mathbf{y}_{k}=\mathbf{h}_{k}\left(\mathbf{x}_{k}, \mathbf{w}_{k}\right),
\end{aligned}
$$

where $\mathbf{y}_{k} \in \mathbb{R}^{n_{z}}$ is the measurement vector at discrete time $k, \mathbf{x}_{k} \in \mathbb{R}^{n_{x}}$ is the state vector and $\mathbf{f}_{k}(\cdot)$ and $\mathbf{h}_{k}(\cdot)$ are arbitrary nonlinear mappings of appropriate dimensions. The noise vectors $\mathbf{v}_{k} \in \mathbb{R}^{n_{v}}, \mathbf{w}_{k} \in \mathbb{R}^{n_{w}}$ and the initial state $\mathbf{x}_{0}$ are assumed mutually independent white processes with arbitrary but known probability density functions (pdfs). We further introduce $\mathbf{X}_{k}=\left[\mathbf{x}_{0}^{\top}, \ldots, \mathbf{x}_{k}^{\top}\right]^{\top}$ and $\mathbf{Y}_{k}=$ $\left[\mathbf{y}_{1}^{\top}, \ldots, \mathbf{y}_{k}^{\top}\right]^{\top}$ which denote the collection of augmented states and measurement vectors up to time $k$. In order to keep the notation simple in the rest of the paper, we introduce the following abbreviations: $f_{k}=p\left(\mathbf{x}_{k} \mid \mathbf{x}_{k-1}\right)$, $g_{k}=p\left(\mathbf{y}_{k} \mid \mathbf{x}_{k}\right)$ and $p_{k}=p\left(\mathbf{Y}_{k}, \mathbf{X}_{k}\right)$.

In nonlinear filtering, one is interested in estimating the current state $\mathbf{x}_{k}$ from the sequence of available noisy measurements $\mathbf{Y}_{k}$. The corresponding estimator is denoted as $\hat{\mathbf{x}}_{k}\left(\mathbf{Y}_{k}\right)$, which is a function of the measurement sequence $\mathbf{Y}_{k}$. The performance of any estimator $\hat{\mathbf{x}}_{k}\left(\mathbf{Y}_{k}\right)$ is commonly measured by the MSE matrix

$$
\mathcal{M}\left(\hat{\mathbf{x}}_{k}\right)=\mathbb{E}\left\{\left(\hat{\mathbf{x}}_{k}\left(\mathbf{Y}_{k}\right)-\mathbf{x}_{k}\right)\left(\hat{\mathbf{x}}_{k}\left(\mathbf{Y}_{k}\right)-\mathbf{x}_{k}\right)^{\top}\right\} .
$$

The idea followed by Reece and Nicholson is to derive a Bayesian Bhattacharyya bound for the MSE matrix of the sequence of estimators $\hat{\mathbf{X}}_{k}\left(\mathbf{Y}_{k}\right)=$ $\left[\hat{\mathbf{x}}_{0}\left(\mathbf{Y}_{k}\right), \ldots, \hat{\mathbf{x}}_{k}\left(\mathbf{Y}_{k}\right)\right]^{\top}$, which is given by

$$
\mathcal{M}\left(\hat{\mathbf{X}}_{k}\right)=\mathbb{E}\left\{\left(\hat{\mathbf{X}}_{k}\left(\mathbf{Y}_{k}\right)-\mathbf{X}_{k}\right)\left(\hat{\mathbf{X}}_{k}\left(\mathbf{Y}_{k}\right)-\mathbf{X}_{k}\right)^{\top}\right\},
$$

This matrix has size $n_{x}(k+1) \times n_{x}(k+1)$ and is growing with time index $k$. The MSE matrix for estimating the state $\mathbf{x}_{k}$ can be found by taking the $n_{x} \times n_{x}$ lower-right submatrix of $\mathcal{M}\left(\hat{\mathbf{X}}_{k}\right)$, which can be expressed mathematically as

$$
\mathcal{M}\left(\hat{\mathbf{x}}_{k}\right)=\mathbf{U} \mathcal{M}\left(\hat{\mathbf{X}}_{k}\right) \mathbf{U}^{\top}
$$

with mapping matrix $\mathbf{U}=\left[\mathbf{0}, \ldots, \mathbf{0}, \mathbf{I}_{n_{x}}\right]$, where $\mathbf{I}_{n_{x}}$ is the $n_{x} \times n_{x}$ identity matrix and $\mathbf{0}$ is a matrix of zeros of appropriate size.

In [6], [7], the so-called Weiss-Weinstein family of bounds on the MSE has been introduced, for which the Bayesian Bhattacharyya bound is a special case. A corresponding Weiss-Weinstein family of lower bounds for the MSE matrix of the state vector sequence $\mathbf{X}_{k}$ can be stated as

$$
\mathcal{M}\left(\hat{\mathbf{X}}_{k}\right) \geq \mathbf{V}^{(k)}\left[\mathbf{J}^{(k)}\right]^{-1} \mathbf{V}^{(k), \top}
$$

with matrix elements

$$
\begin{aligned}
{\left[\mathbf{V}^{(k)}\right]_{i, j} } & =\mathbb{E}\left\{x_{i} \psi_{j}\left(\mathbf{Y}_{k}, \mathbf{X}_{k}\right)\right\} \\
{\left[\mathbf{J}^{(k)}\right]_{i, j} } & =\mathbb{E}\left\{\psi_{i}\left(\mathbf{Y}_{k}, \mathbf{X}_{k}\right) \psi_{j}\left(\mathbf{Y}_{k}, \mathbf{X}_{k}\right)\right\}
\end{aligned}
$$

and where $x_{i}$ denotes the $i$-th element of $\mathbf{X}_{k}=$ $\left[x_{1}, \ldots, x_{(k+1) n_{x}}\right]$. Note, that $\psi_{i}\left(\mathbf{Y}_{k}, \mathbf{X}_{k}\right)$ is the $i$ th element of an $K \times 1$ vector $\boldsymbol{\psi}\left(\mathbf{Y}_{k}, \mathbf{X}_{k}\right)=$ $\left[\psi_{1}\left(\mathbf{Y}_{k}, \mathbf{X}_{k}\right), \ldots, \psi_{K}\left(\mathbf{Y}_{k}, \mathbf{X}_{k}\right)\right]^{\top}$ and $\psi_{i}\left(\mathbf{Y}_{k}, \mathbf{X}_{k}\right)$ has to satisfy for $i=1, \ldots, K$ the following condition

$$
\int \psi_{i}\left(\mathbf{Y}_{k}, \mathbf{X}_{k}\right) p_{k} \mathrm{~d} \mathbf{X}_{k}=0, \quad \forall \mathbf{Y}_{k}
$$

In order for the bound matrix to be full rank, we require that the dimension $K$ of the vector $\psi\left(\mathbf{Y}_{k}, \mathbf{X}_{k}\right)$ be at least $(k+1) n_{x}$ [4]. The $N$-th order vector parameter Bayesian Bhattacharyya bound proposed by Reece and Nicholson [3] 
assumes that $K=(k+1) n_{x}$ and that $\psi_{i}\left(\mathbf{Y}_{k}, \mathbf{X}_{k}\right)$ is given as follows

$$
\psi_{i}\left(\mathbf{Y}_{k}, \mathbf{X}_{k}\right)=\sum_{n=1}^{N} a_{n, i} \cdot \frac{1}{p_{k}} \frac{\partial^{n} p_{k}}{\partial x_{i}^{n}}
$$

$i=1, \ldots,(k+1) n_{x}$, where $a_{n, i}$ are arbitrary real numbers that are chosen such that the bound is maximized. For each state vector $\mathbf{x}_{\xi}$ in the state vector sequence $\mathbf{X}_{k}$, we define a vector $\mathbf{a}_{\xi}=\left[a_{1,1}(\xi), \ldots, a_{N, 1}(\xi), \ldots, a_{1, n_{x}}(\xi), \ldots, a_{N, n_{x}}(\xi)\right]^{\top}$ with mapping $a_{n, m}(\xi)=a_{n,(\xi+1) m}$, and $\xi=0, \ldots, k$, such that $\mathbf{A}_{k}=\left[\mathbf{a}_{0}^{\top}, \mathbf{a}_{1}^{\top}, \ldots, \mathbf{a}_{k}^{\top}\right]^{\top}$. It is assumed further, that the following regularity conditions are fulfilled

- $\quad$ The partial derivatives $\partial^{n} p_{k} / \partial x_{i}^{n}, n=0, \ldots,(N-$ 1 ), are absolutely continuous with respect to $x_{i}$ a.e. $\left\{\mathbf{Y}_{k}, x_{1}, \ldots, x_{i-1}, x_{i+1}, \ldots, x_{(k+1) n_{x}}\right\}$

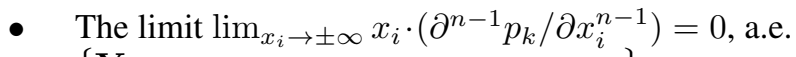
$\left\{\mathbf{Y}_{k}, x_{1}, \ldots, x_{i-1}, x_{i+1}, \ldots, x_{(k+1) n_{x}}\right\}$

- The matrix $\mathbf{J}^{(k)}$ is non-singular,

Then, a corresponding $N$-th order Bayesian Bhattacharyya can be derived, which is given by

$$
\mathcal{M}\left(\hat{\mathbf{X}}_{k}\right) \geq \max _{\mathbf{A}_{k}}\left[\mathbf{V}^{(k)}\left[\mathbf{J}^{(k)}\right]^{-1}\left(\mathbf{V}^{(k)}\right)^{\top}\right],
$$

with block-diagonal mapping matrix

$$
\mathbf{V}^{(k)}=\operatorname{blkdiag}\left(\mathbf{V}_{0}, \mathbf{V}_{1}, \ldots, \mathbf{V}_{k}\right),
$$

where $\mathbf{V}_{\xi}=-\operatorname{diag}\left(\left[a_{1,1}(\xi), \ldots, a_{1, n_{x}}(\xi)\right]\right)$, and information matrix

$$
\mathbf{J}^{(k)} \triangleq\left[\begin{array}{ccc}
\mathbf{J}^{(k)}(0,0) & \cdots & \mathbf{J}^{(k)}(0, k) \\
\vdots & \ddots & \vdots \\
\mathbf{J}^{(k)}(k, 0) & \cdots & \mathbf{J}^{(k)}(k, k)
\end{array}\right]
$$

which is partitioned into blocks $\mathbf{J}^{(k)}(\xi, \eta), \xi, \eta=0, \ldots, k$ each of size $n_{x} \times n_{x}$, and whose $(i, j)$-th element is given by

$$
\begin{aligned}
& {\left[\mathbf{J}^{(k)}(\xi, \eta)\right]_{i, j}=} \\
& \quad \sum_{m=1}^{N} \sum_{n=1}^{N} a_{m, i}(\xi) a_{n, j}(\eta) \cdot \mathbb{E}\left\{\frac{1}{p_{k}^{2}} \frac{\partial^{m} p_{k}}{\partial x_{\xi, i}^{m}} \frac{\partial^{n} p_{k}}{\partial x_{\eta, j}^{n}}\right\} .
\end{aligned}
$$

The expression in (11) denotes the Bayesian Bhattacharyya information matrix for the state sequence $\mathbf{X}_{k}$. The Bayesian Bhattacharyya bound for estimating the current state $\mathbf{x}_{k}$ is obtain by extracting the lower-right $n_{x} \times n_{x}$ partition of the matrix inverse $\left[\mathbf{J}^{(k)}\right]^{-1}$. Since the matrix $\mathbf{J}^{(k)}$ has size $n_{x}(k+1) \times n_{x}(k+1)$ and is growing with time index $k$, the required matrix inversion eventually becomes infeasible for large $k$. In order to keep the computational complexity low, a recursive computation of the inverse of the lower-right partition of the matrix inverse $\left[\mathbf{J}^{(k)}\right]^{-1}$, denoted as Bayesian Bhattacharyya information submatrix $\mathbf{J}_{k}$, is sought after, which is presented next.

\section{Recursive CAlculation of the Bound}

In order to establish a recursion for the information submatrix $\mathbf{J}_{k}$ of the current state $\mathbf{x}_{k}$, the Bayesian Bhattacharyya information matrix $\mathbf{J}^{(k)}$ of the state sequence $\mathbf{X}_{k}$ has to satisfy certain structural properties. These are established in the following Lemmas and Proposition.

A large number of entries on the off-diagonal of the information matrix $\mathbf{J}^{(k)}$ are zero.

Lemma 1. For $\xi \leq k-2$ it holds that

$$
\mathbf{J}^{(k)}(\xi, k)=\mathbf{0} \text {. }
$$

Proof: Due to space limitations, the proof is provided in an accompanying technical report [8].

Further, due to the Markov property of the state $\mathbf{x}_{k}$ many elements in the information matrix remain the same.

Lemma 2. For $\xi, \eta \leq k-2$ it holds that

$$
\mathbf{J}^{(k)}(\xi, \eta)=\mathbf{J}^{(k-1)}(\xi, \eta) .
$$

Proof: See technical report [8].

Similarly, we have

Lemma 3. For $\xi \leq k-2$ it holds that

$$
\mathbf{J}^{(k)}(\xi, k-1)=\mathbf{J}^{(k-1)}(\xi, k-1) .
$$

Proof: See technical report [8].

Proposition 1. The block matrix $\mathbf{J}^{(k)}(k-1, k-1)$ can be decomposed as follows

$$
\begin{aligned}
& \mathbf{J}^{(k)}(k-1, k-1)= \\
& \quad \mathbf{J}^{(k-1)}(k-1, k-1)+\widetilde{\mathbf{J}}^{(k)}(k-1, k-1),
\end{aligned}
$$

where the $(i, j)$-th element of the matrix $\widetilde{\mathbf{J}}^{(k)}(k-1, k-1)$ is given by

$$
\begin{aligned}
& {\left[\widetilde{\mathbf{J}}^{(k)}(k-1, k-1)\right]_{i, j}=\sum_{m=1}^{N} \sum_{n=1}^{N} \sum_{r=0}^{m-1} \sum_{s=0}^{n-1} a_{m, i}(k-1)} \\
& \times a_{n, j}(k-1)\left(\frac{m !}{r !(m-r) !}\right)\left(\frac{n !}{s !(n-s) !}\right) \\
& \times \mathbb{E}\left\{\frac{1}{f_{k}^{2} p_{k-1}^{2}} \frac{\partial^{m-r} f_{k}}{\partial x_{k-1, i}^{m-r}} \frac{\partial^{r} p_{k-1}}{\partial x_{k-1, i}^{r}} \frac{\partial^{n-s} f_{k}}{\partial x_{k-1, j}^{n-s}} \frac{\partial^{s} p_{k-1}}{\partial x_{k-1, j}^{s}}\right\} \cdot
\end{aligned}
$$

Proof: See technical report [8].

With the results of Lemma 1 to Lemma 3 and Proposition 1 , it is possible to partition the matrix $\mathbf{J}^{(k)}$ into a block tri-diagonal form. Subsequent use of the block matrix inversion lemma then gives the desired recursion for the information matrix

$$
\begin{aligned}
\mathbf{J}_{k} & =\mathbf{J}^{(k)}(k, k)-\mathbf{J}^{(k)}(k, k-1) \\
& \times\left[\widetilde{\mathbf{J}}^{(k)}(k-1, k-1)+\mathbf{J}_{k-1}\right]^{-1} \mathbf{J}^{(k)}(k-1, k),
\end{aligned}
$$

see [8] for more details. The corresponding $N$-th order Bayesian Bhattacharyya bound for estimating the current state is then given by

$$
\mathcal{M}\left(\hat{\mathbf{x}}_{k}\right) \geq \max _{\mathbf{A}_{k}}\left[\mathbf{V}_{k}^{\top}\left[\mathbf{J}_{k}\right]^{-1} \mathbf{V}_{k}\right] .
$$




\section{SECOND-ORDER BAYESIAN BHATTACHARYYA BOUND}

The recursive computation of the $N$-th order Bayesian Bhattacharyya information submatrix according to (18) requires the evaluation of the terms $\mathbf{J}^{(k)}(k, k), \mathbf{J}^{(k)}(k-1, k)$ and $\widetilde{\mathbf{J}}^{(k)}(k-1, k-1)$. As this is generally a tedious procedure, computation of terms up to order $N=2$ only have been considered in [3]. We present in (20) only the final, corrected expressions for this case and refer the reader interested in the derivations to the technical report [8]. A comparison with the results obtained in [3] reveal that some terms given in (20) are missing in [3]. In particular, the latter four terms in $\mathbf{J}^{(k)}(k, k)$, see (20a), the latter two terms in $\mathbf{J}^{(k)}(k-1, k)$, see $(20 \mathrm{~b})$ and the latter four terms in $\widetilde{\mathbf{J}}^{(k)}(k-1, k-1)$, see (20c), are missing. These expectations are, in general, not zero, as will be shown in the nonlinear toy example. Remark 1: Closed form expressions for $p_{k-1}$ and its gradient are often not available. In these cases, the expression given in (20) can be written into a different equivalent form by making use of the following identity

$$
\frac{1}{p_{k-1}} \frac{\partial p_{k-1}}{\partial x_{k-1, m}}=\frac{1}{g_{k-1}} \frac{\partial g_{k-1}}{\partial x_{k-1, m}}+\frac{1}{f_{k-1}} \frac{\partial f_{k-1}}{\partial x_{k-1, m}},
$$

see [8] for the details.

Remark 2: It is worth noting that the aforementioned extra terms of (20) are zero in the example presented in [3]. This, however, is a result of the model assumptions and does not hold in general.

The computation of the Bayesian Bhattacharyya bound according to (19) requires to solve an optimization problem with a large number of variables $\mathbf{A}_{k}$. In order to be mathematically more precise, the matrix $\mathbf{J}_{k}$ should be written as a function of $\mathbf{A}_{k}$. Hence, in order to minimize $\mathbf{J}_{k}\left(\mathbf{A}_{k}\right)$ we would need to recalculate $\mathbf{J}_{k-1}\left(\mathbf{A}_{k-1}\right)$ for every time step. Clearly, this prevents (18) being a true recursion. In order to avoid this tedious procedure, the authors of [3] propose to perform the optimization with respect to $\mathbf{a}_{k}$ only (further, they set $a_{1, m}(k)=1$ for all $k$ ). While this approach is computationally effective, it generally yields a suboptimal Bayesian Bhattacharyya bound.

\section{NONLINEAR TOY EXAMPLE}

We consider a 1-D dynamical system with a linear, additive Gaussian process model, such that the transition pdf is given by

$$
p\left(x_{k} \mid x_{k-1}\right)=\frac{1}{\sqrt{2 \pi Q}} \exp \left\{-\frac{\left(x_{k}-x_{k-1}\right)^{2}}{2 Q}\right\},
$$

and a nonlinear, additive Gaussian measurement model, yielding the following likelihood

$$
p\left(y_{k} \mid x_{k}\right)=\frac{1}{\sqrt{2 \pi R}} \exp \left\{-\frac{\left(y_{k}-\gamma \cdot x_{k}^{2}\right)^{2}}{2 R}\right\} .
$$

where $0<\gamma<1$. We further assume that the initial state is Gaussian distributed according to

$$
p\left(x_{0}\right)=\frac{1}{\sqrt{2 \pi P_{0 \mid 0}}} \exp \left\{-\frac{\left(x_{0}-\hat{x}_{0 \mid 0}\right)^{2}}{2 P_{0 \mid 0}}\right\} .
$$

Then, the distribution of the state $x_{k}$ is Gaussian, and given by

$$
p\left(x_{k}\right)=\frac{1}{\sqrt{2 \pi\left(k Q+P_{0 \mid 0}\right)}} \exp \left\{-\frac{\left(x_{k}-\hat{x}_{0 \mid 0}\right)^{2}}{2\left(k Q+P_{0 \mid 0}\right)}\right\} .
$$

For the initial information matrix, we have

$$
J^{(0)}(0,0)=\frac{1}{P_{0 \mid 0}}\left[a_{1}(0)\right]^{2}+\frac{2}{P_{0 \mid 0}^{2}}\left[a_{2}(0)\right]^{2},
$$

and the other terms constituting the recursion are given by

$$
\begin{aligned}
& J^{(k)}(k, k)=\left(\frac{4 \gamma^{2}\left\{\hat{x}_{0 \mid 0}^{2}+\left(k Q+P_{0 \mid 0}\right)\right\}}{R}+\frac{1}{Q}\right)\left[a_{1}(k)\right]^{2} \\
& -\left(\frac{24 \gamma^{2}}{R} \hat{x}_{0 \mid 0}\right) a_{1}(k) a_{2}(k)+\left(\frac{36 \gamma^{2}}{R}+\frac{2}{Q^{2}}\right. \\
& +\frac{32 \gamma^{4}\left\{\hat{x}_{0 \mid 0}^{4}+6 \hat{x}_{0 \mid 0}^{2}\left(k Q+P_{0 \mid 0}\right)+3\left(k Q+P_{0 \mid 0}\right)^{2}\right\}}{R^{2}} \\
& \left.+\frac{16 \gamma^{2}\left\{\hat{x}_{0 \mid 0}^{2}+\left((k-1) Q+P_{0 \mid 0}\right)\right\}}{R Q}\right)\left[a_{2}(k)\right]^{2}, \quad \text { (27a) }
\end{aligned}
$$

$J^{(k)}(k-1, k)=-\frac{1}{Q} a_{1}(k-1) a_{1}(k)+\frac{2}{Q^{2}} a_{2}(k-1) a_{2}(k)$,

$$
\begin{aligned}
& \widetilde{J}^{(k)}(k-1, k-1)=\frac{1}{Q}\left[a_{1}(k-1)\right]^{2} \\
& +\left(\frac{6}{Q^{2}}+\frac{16 \gamma^{2}\left\{\hat{x}_{0 \mid 0}^{2}+\left((k-1) Q+P_{0 \mid 0}\right)\right\}}{R Q}\right)\left[a_{2}(k-1)\right]^{2}
\end{aligned}
$$

The derivation details again can be found in the technical report [8]. The proposed model is quite challenging for any estimator as the likelihood is bi-modal due to the squaring operation. Hence, as time evolves, we will obtain a rather large MSE. The Bayesian Cramér-Rao bound and Bhattacharyya bound are local bounds exploiting the curvature of the $\log$ joint density $p_{k}$, and generally have difficulties in capturing the bi-modality effect. However, one of the advantages compared to other bounds in the WeissWeinstein family is that we obtain closed form expressions that are relatively easy to evaluate.

In the following, we investigate two different parameter sets and compute the Bayesian Bhattacharyya bound (BBLB) based on the methodology presented in Section III and compare it to the expression derived in [3] as well as the Bayesian Cramér-Rao bound (BCRLB). We further compare the bounds to the extended Kalman filter (EKF) and the bootstrap particle filter (PF) with transition pdf as importance density using $N_{\mathrm{pf}}=1000$ particles. The MSE performance is computed for these two filters based on $N_{\text {MC }}=20000$ Monte Carlo runs.

Parameter Set 1: We assume $\hat{x}_{0 \mid 0}=5.85, P_{0 \mid 0}=1$, $Q=1.15, \gamma=0.2, R=7$ and the results are presented in Fig. 1. It can be observed, that due to the choice of an 


$$
\begin{aligned}
& {\left[\mathbf{J}^{(k)}(k, k)\right]_{i, j}=\sum_{m=1}^{2} \sum_{n=1}^{2} a_{m, i}(k) a_{n, j}(k)\left[\mathbb{E}\left\{\frac{1}{g_{k}^{2}} \frac{\partial^{m} g_{k}}{\partial x_{k, i}^{m}} \frac{\partial^{n} g_{k}}{\partial x_{k, j}^{n}}\right\}+\mathbb{E}\left\{\frac{1}{f_{k}^{2}} \frac{\partial^{m} f_{k}}{\partial x_{k, i}^{m}} \frac{\partial^{n} f_{k}}{\partial x_{k, j}^{n}}\right\}\right]+4 a_{2, i}(k) a_{2, j}(k) \mathbb{E}\left\{\frac{1}{g_{k}^{2} f_{k}^{2}} \frac{\partial g_{k}}{\partial x_{k, i}} \frac{\partial f_{k}}{\partial x_{k, i}} \frac{\partial g_{k}}{\partial x_{k, j}} \frac{\partial f_{k}}{\partial x_{k, j}}\right\}} \\
& +2 a_{1, i}(k) a_{2, j}(k) \mathbb{E}\left\{\frac{1}{g_{k}^{2} f_{k}} \frac{\partial g_{k}}{\partial x_{k, i}} \frac{\partial f_{k}}{\partial x_{k, j}} \frac{\partial g_{k}}{\partial x_{k, j}}\right\}+2 a_{2, i}(k) a_{1, j}(k) \mathbb{E}\left\{\frac{1}{g_{k}^{2} f_{k}} \frac{\partial g_{k}}{\partial x_{k, i}} \frac{\partial f_{k}}{\partial x_{k, i}} \frac{\partial g_{k}}{\partial x_{k, j}}\right\}+2 a_{2, i}(k) a_{2, j}(k) \mathbb{E}\left\{\frac{1}{g_{k}^{2} f_{k}} \frac{\partial g_{k}}{\partial x_{k, i}} \frac{\partial f_{k}}{\partial x_{k, i}} \frac{\partial^{2} g_{k}}{\partial x_{k, j}^{2}}\right\} \\
& +2 a_{2, i}(k) a_{2, j}(k) \mathbb{E}\left\{\frac{1}{g_{k}^{2} f_{k}} \frac{\partial^{2} g_{k}}{\partial x_{k, i}^{2}} \frac{\partial g_{k}}{\partial x_{k, j}} \frac{\partial f_{k}}{\partial x_{k, j}}\right\} \\
& \quad\left[\mathbf{J}^{(k)}(k-1, k)\right]_{i, j}=\sum_{m=1}^{2} \sum_{n=1}^{2} a_{m, i}(k-1) a_{n, j}(k) \mathbb{E}\left\{\frac{1}{f_{k}^{2}} \frac{\partial^{m} f_{k}}{\partial x_{k-1, i}^{m}} \frac{\partial^{n} f_{k}}{\partial x_{k, j}^{n}}\right\}+2 a_{2, i}(k-1) a_{1, j}(k) \mathbb{E}\left\{\frac{1}{f_{k}^{2} p_{k-1}} \frac{\partial f_{k}}{\partial x_{k-1, i}} \frac{\partial p_{k-1}}{\partial x_{k-1, i}} \frac{\partial f_{k}}{\partial x_{k, j}}\right\} \\
& \quad+2 a_{2, i}(k-1) a_{2, j}(k) \mathbb{E}\left\{\frac{1}{f_{k}^{2} p_{k-1}} \frac{\partial f_{k}}{\partial x_{k-1, i}} \frac{\partial p_{k-1}}{\partial x_{k-1, i}} \frac{\partial^{2} f_{k}}{\partial x_{k, j}^{2}}\right\}=\left[\mathbf{J}^{(k)}(k, k-1)\right]_{j, i}, \\
& \quad+4 a_{2, i}(k-1) a_{2, j}(k-1) \mathbb{E}\left\{\frac{1}{f_{k}^{2} p_{k-1}^{2}} \frac{\partial f_{k}}{\partial x_{k-1, i}} \frac{\partial p_{k-1}}{\partial x_{k-1, i}} \frac{\partial f_{k}}{\partial x_{k-1, j}} \frac{\partial p_{k-1}}{\partial x_{k-1, j}}\right\}+2 a_{1, i}(k-1) a_{2, j}(k-1) \mathbb{E}\left\{\frac{1}{f_{k}^{2} p_{k-1}} \frac{\partial f_{k}}{\partial x_{k-1, i}} \frac{\partial f_{k}}{\partial x_{k-1, j}} \frac{\partial p_{k-1}}{\partial x_{k-1, j}}\right\} \\
& \quad+2 a_{2, i}(k-1) a_{1, j}(k-1) \mathbb{E}\left\{\frac{1}{f_{k}^{2} p_{k-1}} \frac{\partial f_{k}}{\partial x_{k-1, i}} \frac{\partial p_{k-1}}{\partial x_{k-1, i}} \frac{\partial f_{k}}{\partial x_{k-1, j}}\right\}+2 a_{2, i}(k-1) a_{2, j}(k-1) \mathbb{E}\left\{\frac{1}{f_{k}^{2} p_{k-1}} \frac{\partial^{2} f_{k}}{\partial x_{k-1, i}^{2}} \frac{\partial f_{k}}{\partial x_{k-1, j}} \frac{\partial p_{k-1}}{\partial x_{k-1, j}}\right\} \\
& \quad+2 a_{2, i}(k-1) a_{2, j}(k-1) \mathbb{E}\left\{\frac{1}{f_{k}^{2} p_{k-1}} \frac{\partial f_{k}}{\partial x_{k-1, i}} \frac{\partial p_{k-1}}{\partial x_{k-1, i}} \frac{\partial^{2} f_{k}}{\partial x_{k-1, j}^{2}}\right\} \cdot
\end{aligned}
$$

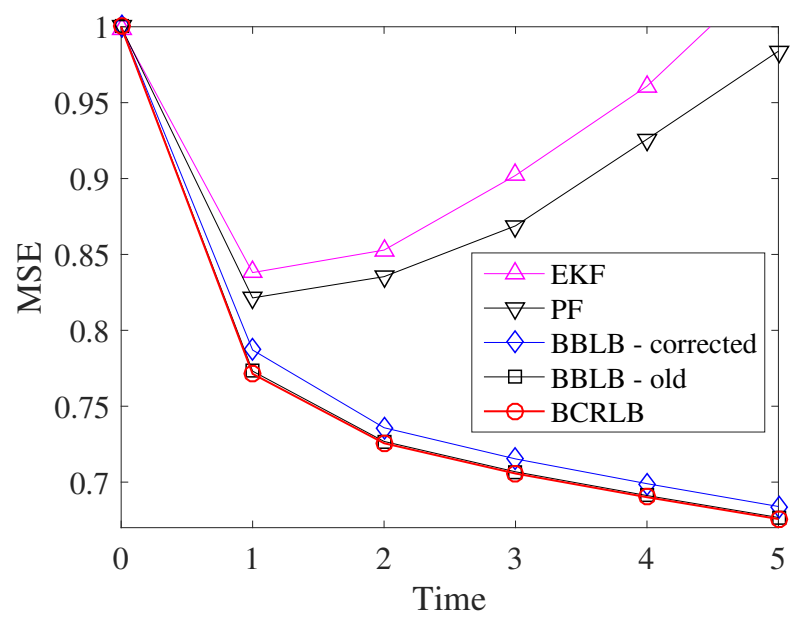

Figure 1. MSE performance vs. time steps for parameter set 1.

initial value relatively far away from zero, the estimator performance and the corresponding bounds are close together. As time evolves, however, the MSE of the two estimators increase which the Bayesian Bhattacharyya and CramérRao bounds cannot capture. It can be further observed that the corrected expression for the Bayesian Bhattacharyya bound is significantly different to the expression proposed in [3], which is similar to the Bayesian Cramér-Rao bound.

Parameter Set 2: We assume $\hat{x}_{0 \mid 0}=1, P_{0 \mid 0}=0.05$, $Q=0.1, \gamma=0.2, R=0.04$ and the results are presented in Fig. 2. Again, the bounds can follow the MSE curves of the estimators in the beginning, but then depart leaving a relatively large gap between estimator performance and corresponding lower bounds. The corrected Bayesian Bhattacharyya bound is again the tightest bound in this setting.

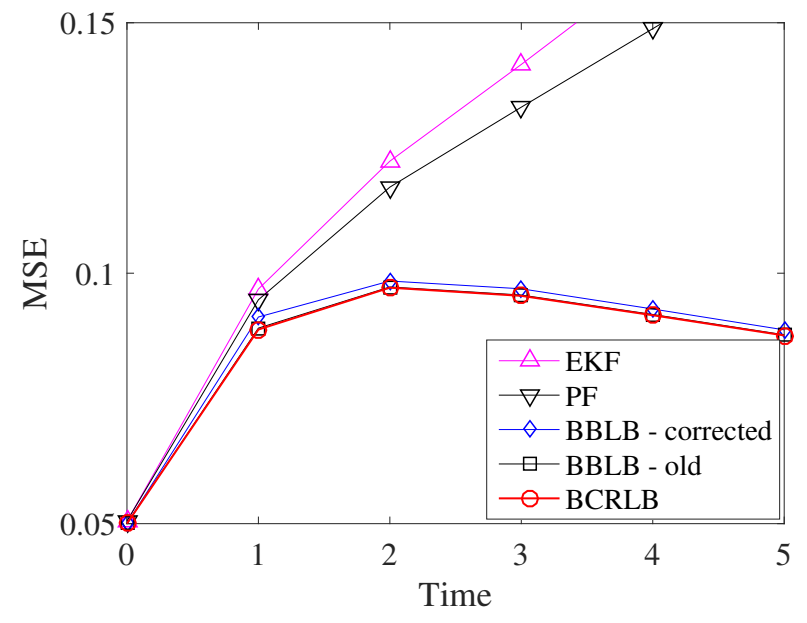

Figure 2. MSE performance vs. time steps for parameter set 2 .

\section{CONCLUSion}

We have presented corrected expressions for a second order Bayesian Bhattacharyya bound that was previously proposed by Reece and Nicholson. A nonlinear toy example is used where the corrected bound is significantly different to the old, incorrect formulation. Future work will include the derivation of Bayesian Bhattacharyya bounds that do not require the complicated optimization procedure of the free variables.

\section{ACKNOWLEDGMENT}

This work was supported in part by the Excellence Center at Linköping and Lund in Information Technology (ELLIIT). 


\section{REFERENCES}

[1] P. Tichavský, C. H. Muravchik, and A. Nehorai, "Posterior CramérRao bounds for discrete-time nonlinear filtering," IEEE Trans. Signal Process., vol. 46, no. 5, pp. 1386-1396, May 1998.

[2] I. Rapoport and Y. Oshman, "Recursive Weiss-Weinstein lower bounds for discrete-time nonlinear filtering," in 43rd IEEE Conference on Decision and Control $(C D C)$, vol. 3, Atlantis, Paradise Island, Bahamas, Dec. 2004, pp. 2662-2667.

[3] S. Reece and D. Nicholson, "Tighter alternatives to the CramérRao lower bound for discrete-time filtering," in 8th International Conference on Information Fusion, vol. 1. Philadelphia, PA, USA, Jul. 2005, pp. 1-6.

[4] H. L. van Trees and K. L. Bell, Eds., Bayesian Bounds for Parameter Estimation and Nonlinear Filtering/Tracking. Piscataway, NJ, USA: Wiley-IEEE Press, 2007.

[5] C. Fritsche, E. Özkan, L. Svennson, and F. Gustafsson, "A fresh look at Bayesian Cramér-Rao bounds for discrete-time nonlinear filtering," in Proc. of 17th International Conference on Information Fusion, Salamanca, Spain, Jul. 2014, pp. 1-7.

[6] A. Weiss and E. Weinstein, "A lower bound on the mean-square error in random parameter estimation," IEEE Trans. Inf. Theory, vol. 31, no. 5, pp. 680-682, Sept. 1985.

[7] E. Weinstein and A. J. Weiss, "Lower bounds on the mean square estimation error," Proceedings of the IEEE, vol. 73, no. 9, pp. 14331434, Sept. 1985.

[8] C. Fritsche, "Derivation of a Bayesian Bhattacharyya bound for discrete-time filtering," Linköping University, Linköping, Sweden, Tech. Rep. LiTH-ISY-R-3099, Jun. 2017. [Online]. Available: http://www.control.isy.liu.se/publications/ 